\title{
\begin{tabular}{l|l} 
Mitraries & DSpace@MIT
\end{tabular}
}

\author{
MIT Open Access Articles
}

\section{Fermi-Energy-Dependent Structural Deformation of Chiral Single-Wall Carbon Nanotubes}

The MIT Faculty has made this article openly available. Please share how this access benefits you. Your story matters.

Citation: Vieira, Bruno G.MM., Eduardo B. Barros, Daniel G. Vercosa, Georgy Samsonidze, Antonio G. Souza Filho, and Mildred S. Dresselhaus. Fermi-Energy-Dependent Structural Deformation of Chiral Single-Wall Carbon Nanotubes. Phys. Rev. Applied 2, 014006 (2014). (c) 2014 American Physical Society

As Published: http://dx.doi.org/10.1103/PhysRevApplied.2.014006

Publisher: American Physical Society

Persistent URL: http://hdl.handle.net/1721.1/88477

Version: Final published version: final published article, as it appeared in a journal, conference proceedings, or other formally published context

Terms of Use: Article is made available in accordance with the publisher's policy and may be subject to US copyright law. Please refer to the publisher's site for terms of use. 


\title{
Fermi-Energy-Dependent Structural Deformation of Chiral Single-Wall Carbon Nanotubes
}

\author{
Bruno G. M. Vieira, ${ }^{1, *}$ Eduardo B. Barros ${ }^{1,2, \dagger}$ Daniel G. Vercosa, ${ }^{3,1}$ Georgy Samsonidze, ${ }^{4}$ \\ Antonio G. Souza Filho, ${ }^{1}$ and Mildred S. Dresselhaus ${ }^{2,5}$ \\ ${ }^{1}$ Departamento de Física, Universidade Federal do Ceará, Fortaleza, Ceará, 60455-760, Brazil \\ ${ }^{2}$ Department of Physics, Massachusetts Institute of Technology, Cambridge, Massachusetts 02139, USA \\ ${ }^{3}$ Electrical and Computer Engineering Department, Rice University, Houston, Texas 77251, USA \\ ${ }^{4}$ Research and Technology Center, Robert Bosch LLC, Cambridge, Massachusetts 02142, USA \\ ${ }^{5}$ Department of Electrical Engineering and Computer Science, Massachusetts Institute of Technology, \\ Cambridge, Massachusetts 02139, USA
}

(Received 25 April 2014; published 22 July 2014)

\begin{abstract}
In this work, we use an extended tight-binding approach for calculating the Fermi-energy dependence of the structural deformation of chiral single-wall carbon nanotubes (SWNTs). We show that, in general, nanotube strains occur in such a way as to avoid a net charge from being accumulated on the nanotube. We also investigate the effect of the Fermi-energy-induced strains on the electronic structure of SWNTs, showing that the optical transition energies change by up to $0.5 \mathrm{eV}$ due to the induced strains and that this change is nearly independent of how the nanotube is deformed. Finally, we also consider the contribution of the electron-electron Coulomb repulsion to the total energy by using an effective regularized potential energy model. We show that the inclusion of the Coulomb repulsion leads to larger strains and smaller net charges transferred to the nanotube.
\end{abstract}

DOI: 10.1103/PhysRevApplied.2.014006

The nanomechanical actuation of carbon nanotubes can be controlled by using different approaches, each relying on a different mechanism for the coupling between the nanotube mechanical properties and some other controllable parameter. Modeling the nanotube nanomechanical responses to external stimuli is usually a difficult task which depends strongly on the processes involved and the type of stimulus used. For example, a nanoelectromechanical actuator works by tuning nanotube properties through an applied external electric field. However, an electric field can affect the nanotube in several different ways, which usually cannot be captured within a simple unique model. For this reason, the most common approach is to select the stimulus process which seems to be most relevant for the particular application and to use it to model the nanotube response. For example, Witkamp, Poot, and van der Zant [1] consider a direct electrostatic (capacitive) force between a gate and a suspended multiwall carbon nanotube to explain the actuation properties observed in their devices. Indeed, there are plenty of devices based on this electrostatic approach, such as the nanotweezers produced by Kim and Lieber [2], the nanobalance developed by Poncharal et al. [3], the single-molecule torsional pendulum proposed by Meyer, Paillet, and Roth [4], and the nanotube oscillator first proposed by Sazonova et al. [5] and further developed by Peng et al. [6]. However, although the electrostatic model could successfully explain the observed results, it is

\footnotetext{
*bruno@fisica.ufc.br

†ebarros@fisica.ufc.br
}

by no means a general model and could not be applied in geometries for which the nanotube is not suspended above the gate.

Another way that a nanoelectromechanical actuator may function is by a quantum-mechanical mechanism based on the change in the lattice parameters in the presence of a charge. This process has been extensively investigated by different authors using theoretical [7-11] and experimental [11-15] techniques for both graphene and carbon nanotubes. In such cases, the usual way to model the nanomechanical actuator mechanism is to include an extra charge (positive or negative) transferred to the nanotube and to optimize the carbon nanotube structure in order to balance the increased electronic energy $[9,10]$. Although this procedure can give interesting insights into the nanomechanical actuation of the carbon nanotubes, it is not the most appropriate to describe the actual mechanism of an electromechanical actuator but is rather a more appropriate way for describing a chemical actuator, for which the amount of charge exchanged by the doping molecule and the nanotube is defined by the chemistry of their interaction and by the concentration of the dopant molecules.

A usual electromechanical actuator functions by contacting the nanotube with a charge reservoir, which can be either a metal (in a field-effect-transistor-like device) or an electrolyte (for electrochemical devices), and an electric field is applied in order to control the flow of charge to or from the nanotube. Although the net result of the electric field is indeed a charge transfer between the reservoir and the nanotube, we will show here that the nanomechanical 
response in such systems cannot be understood simply in terms of the net charge acquired by the nanotube. The reason for this difference is that, in the case of an electromechanical device, the charge is not fixed but is determined by the chemical potential at the interface between the nanomaterial and the charge reservoir.

$A b$ initio modeling of the actuation mechanism for such devices is hindered by the challenge of making an adequate description of the charge reservoir. To avoid this problem, we propose that the effect of the charge reservoir can be described by its effective result, which is to determine the Fermi energy of the nanotube. Once the Fermi energy is defined, the total energy of the nanotube can then be minimized for each value of Fermi energy and the nanotube deformations can then be obtained.

In this work, we calculate the dependence of the axial, radial, and torsional strains on the Fermi energy $(\mu)$ of single-wall carbon nanotubes by using an extended tightbinding model. In a previous work [16], we demonstrate that chiral nanotubes are intrinsically twisted [17] (or naturally torsioned, as it is also called $[16,18])$, due to a torsional instability. This intrinsic twist is explained in terms of an electronically driven stress which causes the nanotube to twist itself in order to relax into a lower energy configuration [16]. This process indicates a coupling between the nanotube structure and its electronic population. The emergence of a natural torsion in chiral carbon nanotubes is further verified by molecular dynamics and by a density-functional tight binding approach [18]. However, in the later work the explicit influence of the electronic energy to the total carbon nanotube energy is disregarded. Furthermore, similar effects are predicted for $\mathrm{MoS}_{2}$ nanotubes and for bent graphene, indicating that the presence of a natural torsion is an intrinsic characteristic of chiral nanotubes $[19,20]$. We in the present work improve the extended tight-binding approach used by Vercosa et al. [16] by considering that the nanotube electronic states are populated according to the Fermi-Dirac distribution with a given electronic temperature $T_{\mathrm{el}}$ and a chemical potential $\mu$ (in the present work, the terms chemical potential and Fermi energy are used interchangeably so that $E_{F}=\mu$ ). The total electronic energy of the nanotube is obtained by summing the energies of the populated electronic states, while the total nanotube energy is given by the sum of the total electronic energy and the energy associated with the ionic repulsion between the constituent carbon atoms. Ionic repulsion energies between up to the seventh-nearest neighbors as a function of the interatomic distances are used [21]. The structural optimization is performed within the adiabatic approximation, such that the total energy is evaluated at the relaxed atomic positions. This approach disregards the effects of phonon contributions to the total energy. This assumption is justified by the fact that the phonon energies are independent of the Fermi energy, thus contributing only to defining the unstrained configuration, and to the fact that the electronic energies involved in the effects studied here will be much larger than the thermal energy of electrons $k_{B} T_{\mathrm{el}}$ at room temperature. Finally, we discuss the effects of the electron-electron Coulomb repulsion on the total energy and how this repulsion affects the nanotube deformations.

For the band-structure calculations, we use an extended tight-binding model [22] which considers the $2 s, 2 p_{x}, 2 p_{y}$, and $2 p_{z}$ atomic orbitals, thus enabling the rehybridization of the $\sigma$ and $\pi$ orbitals and the inclusion of curvature effects. In addition, the elements of the Hamiltonian and overlap matrices are calculated by using interactions up to the seventh-nearest neighbors (long-range atomic interactions). The tight-binding parameters are determined as functions of the interatomic distances, following the scheme based on the density functional theory and developed by Porezag et al. [21]. Moreover, this method uses the geometry of the nanotube directly, without the need of using the zonefolding technique, and makes use of the full symmetry of the structure by adopting the angular-helical representation of the unit cell $[22,23]$.

The optimization process is accomplished numerically by using a simple direct-search method, known as coordinate search, grid search, or compass search $[24,25]$. The method is comprised of searching the immediate surroundings of an initial point in the parameter space to be optimized by using a predefined initial step length for each parameter. The point in the configuration space which yields the greatest reduction in the total energy is chosen for the next step. If none of the surrounding configurations yield a reduction of the total energy, the step lengths are scaled down. The process is repeated until the desired accuracy is obtained. Further details of the optimization process can be obtained in Refs. [16,22].

We show that the three types of strain (axial, radial, and torsional) are equally relevant to the relaxation process and the changes in the optical transition energies are nearly independent of how the nanotube is deformed. Also, these findings draw attention to the fact that the torsional strain of the nanotube and its electronic properties can be controlled by applying a gate voltage to the nanotube, which opens up a wide range of possibilities for technological applications in the field of nanoelectromechanical devices.

To ensure a more realistic analysis, we consider the electrons to be at room temperature $\left(T_{\mathrm{el}}=300 \mathrm{~K}\right)$, while the Fermi energy $\mu$ was varied from -2.0 to $2.0 \mathrm{eV}$ in steps of $0.1 \mathrm{eV}$, where $\mu=0$ corresponds to the case where the Fermi energy is in the middle of the electronic band gap, and thus no net charge is present in the nanotube. For each value of $\mu$, the nanotube structure is optimized, and, from the values of the optimized parameters, it is possible to calculate how the torsional, radial, and axial strains depend on the Fermi energy.

Figures 1(a)-1(c) show the values of the torsional strain $\varepsilon_{\tau}$ (TS) for the optimized structure as a function of the 
Fermi energy $\mu$. As can be seen, there is a great variation in $\varepsilon_{\tau}$ for all of the three single-wall carbon nanotubes (SWNTs) studied here: $(8,7),(9,7)$, and $(9,6)$. It can also be noted that the torsional strain shows an abrupt change whenever $\mu$ is close to reaching one of the nanotube subband extrema. This phenomenon will be discussed in detail later in the present work. It is also important to comment on the similarities and differences between the three curves of Figs. 1(a)-1(c). First of all, it can be seen that, as the Fermi energy reaches the first electronic subband extremum, the $(8,7)$ nanotube suffers a small negative torsional strain at first, and afterwards the direction of the torsional strain reverses itself abruptly, leading to a torsional strain on the order of $0.75 \%$ at $\mu \approx \pm 1 \mathrm{eV}$. As the second electronic subband is reached, the effect reverses itself again, leading to negative torsions. For the $(9,7)$ nanotube, the effect is quite similar but shows one striking difference: The direction of the torsional strain is opposite; i.e., when the first subband is reached, the torsional strain is initially positive and then reverses towards negative values, and so on for the second subband. These opposite behaviors between the $S 1$ and $S 2$ SWNTs is similar to the behavior of the band gap of SWNTs under uniaxial and torsional strains, as verified by Yang and Han [26]. For the $(9,6)$ SWNT, which is metallic, a relevant variation of its torsion is also observed only when $\mu$ reaches the $E_{11}$ subband.
It is relevant to point out that similar calculations were performed in this work for a large number of SWNTs (not shown here) and that a clear pattern could be observed. In fact, all semiconducting tubes of type $S 1$, for which $\bmod (n-m, 3)=1$, show similar behaviors as that observed for the $(8,7)$ nanotube shown in Fig. 1(a), while all $S 2$ nanotubes $[\bmod (n-m, 3)=2]$ behave as the $(9,7)$ nanotube, and the metallic $M 0[\bmod (n-m, 3)=0]$ nanotubes behave as the $(9,6)$ nanotube. Similar qualitative behaviors are obtained for nanotubes of up to $1.7 \mathrm{~nm}$.

In Figs. 1(d)-1(i), we show the axial strain $\varepsilon_{T}$ (AS) and the radial strain $\varepsilon_{R}$ (RS) as a function of the Fermi energy $\mu$. It can be seen that, for all three nanotubes $[(8,7),(9,7)$, and $(9,6)]$, there is a clear tendency for compression in both the axial and radial directions with increasing $|\mu|$. Likewise, in the case of the torsional strain, there is a change of behavior whenever the value of $\mu$ reaches one of the nanotube subband extrema. Furthermore, another interesting feature about this dependence of $\varepsilon_{T}$ and $\varepsilon_{R}$ on $\mu$ is the fact that, although there is an overall tendency for nanotube compression, which is nearly symmetric for positive and negative values of $\mu$, the behavior of the axial and the radial strains as $\mu$ reaches a valence subband is opposite to that observed when $\mu$ reaches a conduction subband. This behavior as a function of $\mu$ is contrary to what is observed for the torsional strains, which, as discussed above, show a

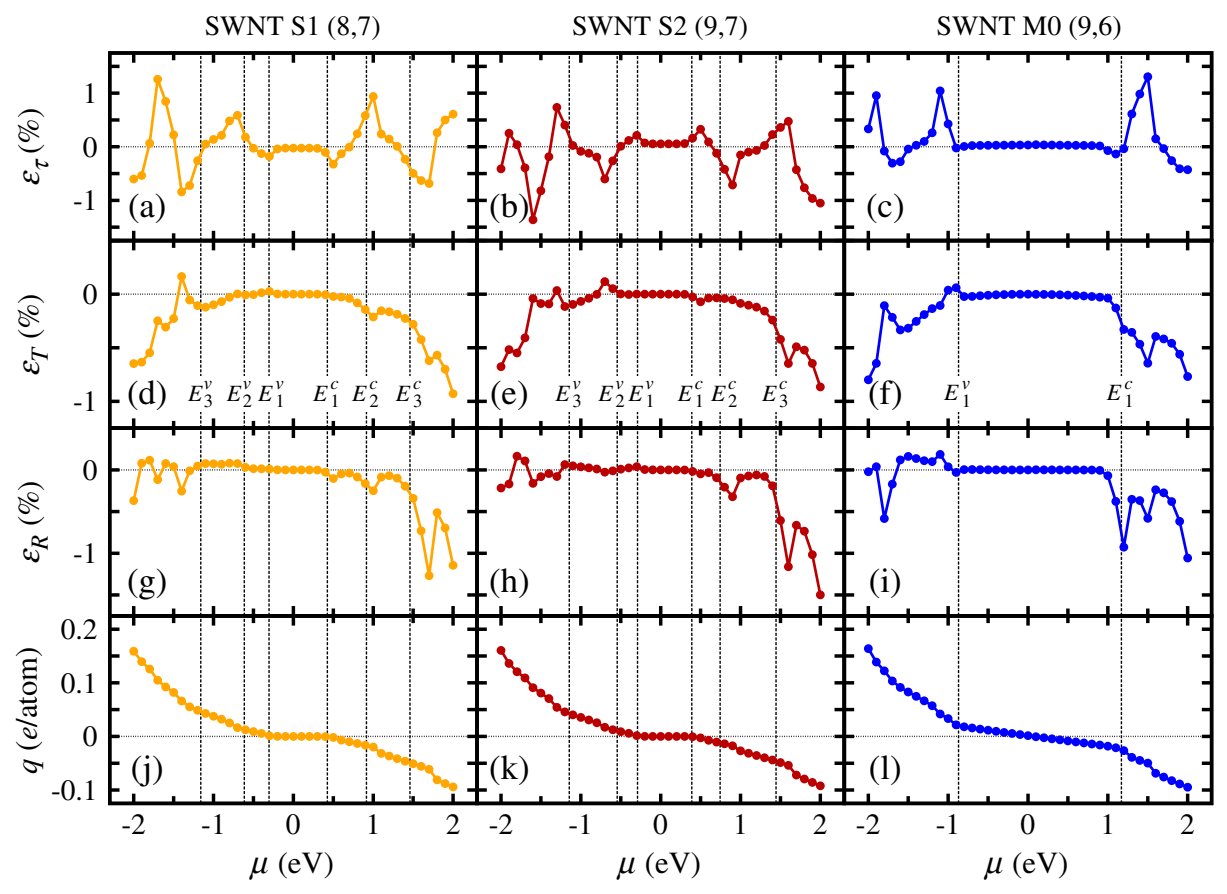

FIG. 1. Torsional, axial, and radial strains as a function of the Fermi energy $(\mu)$ for the type-1 $[\bmod (n-m, 3)=1]$ and type-2 $[\bmod (n-m, 3)=2]$ semiconducting SWNTs $(8,7)$ and $(9,7)$, respectively, and for the metallic (type-0) SWNT (9,6). The resulting injected charge $q$ for each value of $\mu$ is also provided for a more complete analysis, and this charge is in units of the elementary charge $e$ per carbon atom in the SWNT structure. Moreover, the approximate values for the valence subband maximum $\left(E_{1}^{v}, E_{2}^{v}\right.$, and $\left.E_{3}^{v}\right)$ and for the conduction subband minimum $\left(E_{1}^{c}, E_{2}^{c}\right.$, and $\left.E_{3}^{c}\right)$ on each nanotube are shown by the vertical lines as guides to the eye. 
qualitatively symmetric behavior for the valence and conduction bands; see Figs. 1(a)-1(c).

It is now interesting to discuss how these electronically driven axial, radial, and torsional strains can affect the carbon nanotube properties. To accomplish this, an optimization of the structural parameters is performed for each value of $\mu$ in the following four cases: all optimized (all opt)—where all structural parameters are optimized; torsional strain optimized (TS opt)—where axial and radial strains are kept constant; axial strain optimized (AS opt) where torsional and radial strains are kept constant; and radial strain optimized (RS opt) — where axial and torsional strains are kept constant. In the present work, we will focus on the dependence of the optical transition energies on the Fermi energy $\mu$.

In Fig. (2), the energies for the two first optical transition energies $E_{11}$ and $E_{22}$ of the $(8,7)$ semiconducting nanotube as a function of $\mu$ are shown as black circles for the all-opt case. For comparison, we also show the results obtained for the TS-opt, AS-opt, and RS-opt cases (red diamonds, blue triangles, and green squares, respectively). It can be seen that, for all these cases, the transition energies undergo a large variation as the Fermi energy changes, although the variations of the $E_{i i}$ values are only slightly smaller for the AS-opt and RS-opt cases. These results indicate that these effects on the optical transition energies are a general result and that these results are nearly independent of the specific

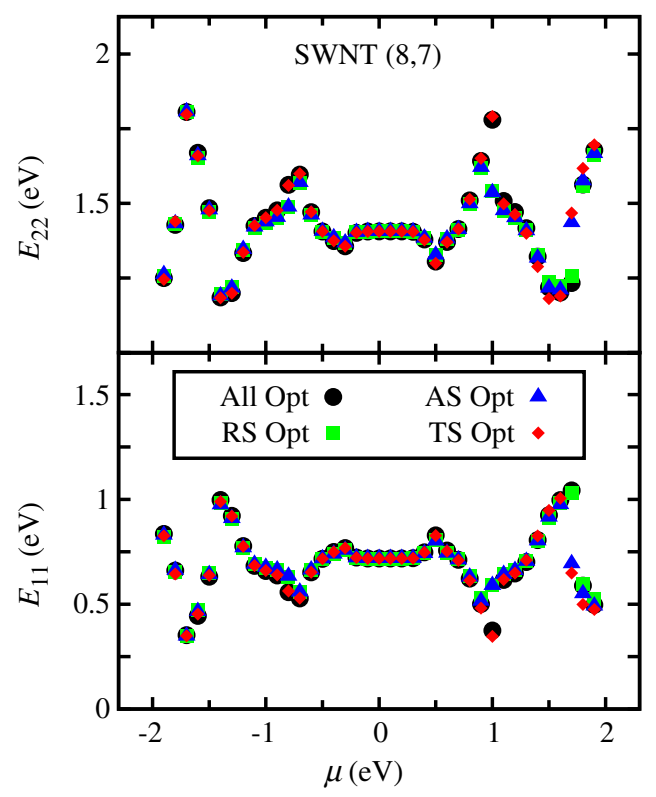

FIG. 2. First $E_{11}$ and second $E_{22}$ optical transition energies of the SWNT $(8,7)$ as a function of the Fermi energy $\mu$ calculated for a full structural optimization (black circles) and for the case where only one of the strains is optimized, while the others are fixed at their optimized values for $\mu=0.0 \mathrm{eV}$, following the sequence: (a) torsional strain optimization (red diamonds); (b) axial strain optimization (blue triangles); (c) radial strain optimization (green squares). way in which the nanotube deformation occurs, be it by radial, axial, or torsional deformations or a combination of the three.

We would next like to clarify the physical process behind the dependence of the torsional strain on the Fermi energy and its consequences on the carbon nanotube structure. For this, we show in Fig. (3) the complete band structure of the $(8,7)$ nanotube for $\mu=0.0,0.5$, and $1.0 \mathrm{eV}$ (left, middle, and right panels, respectively) in the reduced Brillouin zone (BZ) scheme. Within this scheme, the wave vector $\mathbf{k}$ is defined for the pure translational vector $\mathbf{T}$, taking into account the pure translational symmetry of the nanotube [23]. However, as the torsional strain is being considered in this work, this pure translational symmetry is broken in the strained structures. Nevertheless, although the reduced-BZ scheme is not strictly appropriate to represent the band structure of these distorted materials, we choose to use this scheme in Fig. (3) only to allow for a clearer physical discussion of these results, while the extended-BZ scheme, which is defined by using the helical nanotube symmetry [23], is used for all the actual calculations reported here.

First of all, it can be seen that most of the changes in $E(k)$ happen at the band extrema (which are highlighted in each panel). In order to understand the effects of the Fermi energy, we focus our attention on the bands in which the $E_{11}$ and $E_{22}$ optical transitions are located, shown as the highlighted lines in Fig. 3. It can be seen that, as $\mu$ approaches the first unoccupied subband, the carbon nanotube tends to twist itself in order to avoid the occupation of this band, since this band occupation would invariably

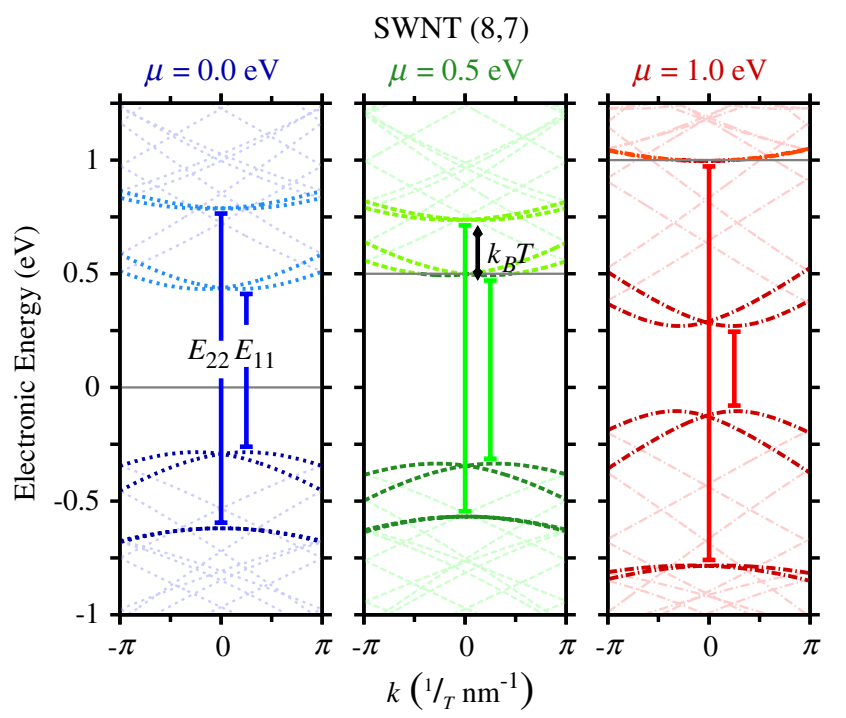

FIG. 3. The band structure $E(k)$ of the $(8,7)$ SWNT for $\mu=0.0$ (left), 0.5 (middle), and $1.0 \mathrm{eV}$ (right). In each panel, the band maxima and minima corresponding to the $E_{11}$ and $E_{22}$ transition energies are highlighted for clarity. The wave number $k$ is given in units of $(1 / T) \mathrm{nm}^{-1}$, where $T$ is the magnitude of the translational vector $\mathbf{T}$ along the axial direction of the SWNT [22]. 
increase the total electronic energy of the SWNT. This twist causes the $E_{11}$ gap to become slightly larger, as is seen also in Fig. 2, but at the same time causes the $E_{22}$ transition energy to decrease. At $\mu=0.5 \mathrm{eV}, E_{11}$ becomes close to $E_{22}$ so that it is no longer possible to prevent the occupation of both conduction subbands, and thus it becomes energetically more favorable for the nanotube to twist itself in the opposite direction so that the $E_{11}$ gap decreases. This happens since the density of states has a peak at the conduction band minimum. Therefore, in order to minimize the electronic energy, the nanotube deformation is now in the direction that takes the band minimum towards the lowest energy possible, decreasing the nanotube band gap and increasing the $E_{22}$ gap so that the second subband remains unoccupied. As $\mu$ is increased and other subbands are reached, a similar process takes place, thereby explaining the recurrent behavior observed for both the dependence of the torsional strain and the $E_{i i}$ transition energies.

One of the shortcomings of the tight-binding model used in this work is that it does not explicitly consider the Coulomb repulsion between the excess charges injected into the nanotube. This repulsion can have important effects on the dependence of the nanotube deformations on the Fermi energy $(\mu)$. Including this repulsion energy is not trivial in twisted systems, for which there is no pure translational symmetry. In order to evaluate this repulsive energy effect qualitatively, we have considered a simple 1D model to describe the Coulomb repulsion energy term in the total energy of the structure. We briefly describe this model below.

Consider that the nanotube is a 1D wire of length $L$ and that the total charge $Q$ is uniformly distributed along the nanotube length; i.e., the wire has a constant linear charge density. Taking into account that the nanotubes have a finite nonzero diameter, we base the calculations on the following regularized $1 \mathrm{D}$ potential $[27,28]$ :

$$
V(z)=\frac{1}{4 \pi \epsilon} \frac{q}{\left|z-z_{0}\right|+d},
$$

in which $z$ is the coordinate of this $1 \mathrm{D}$ wire along the nanotube axis and $d$ is the diameter of the SWNT, $\epsilon$ is an effective dielectric constant, and $q$ is a unit charge located at $z=z_{0}$ in Eq. (1).

Considering the potential in Eq. (1), the total repulsion energy $E_{\text {total }}^{(\text {rep })}$ can be calculated as

$$
E_{\text {total }}^{(\mathrm{rep})}(\lambda, L, d)=\frac{\lambda^{2} L}{4 \pi \epsilon}\left[-1+\left(1+\frac{d}{L}\right) \ln \left(1+\frac{L}{d}\right)\right],
$$

where $\lambda$ is the constant linear charge density along the tube. Because of the long-range Coulomb interaction, we consider that the nanotube length is given by $L=N_{\text {cells }} T$, where $N_{\text {cells }}$ is the number of translational unit cells, which for the $(8,7)$ carbon nanotube analyzed in this work is
$N_{\text {cells }}=18$, corresponding to a length of approximately $100 \mathrm{~nm}$. Different values of $N_{\text {cells }}$ lead to different calculated deformations; however, the results are qualitatively consistent with each other. By analyzing Eq. (2), it can be verified that, if the net charge of the nanotube is fixed, the contribution of the electron-electron Coulomb interaction would, in principle, create a stress towards the expansion in both the axial and radial directions and would not have any significant influence on the torsional degree of freedom.

Figure 4 shows the behavior of the radial, axial, and torsional strains as a function of $\mu$ for the $(8,7)$ SWNT when the Coulomb interaction is considered. This figure also provides previous results obtained without the Coulomb repulsion (blue points and lines) in order to enable comparisons, and they are labeled as $\epsilon \rightarrow \infty$. The calculations are performed for different values of $\epsilon$ in order to simulate a static screening due to the intrinsic permittivity of the nanotube and the environmental influence. The intrinsic dielectric constant $\epsilon_{\mathrm{NT}}$ for nanotubes and for graphene is approximately $4 \epsilon_{0}$ (with $\epsilon_{0}$ being the vacuum permittivity) [29]. For this reason, in the following analysis, we focus on what happens for $\epsilon=4 \epsilon_{0}$. In this case, it can be noted that, although a simple analysis would lead us to believe that the Coulomb repulsion would produce a tendency for expansion in both the axial and the radial directions, while not affecting the torsional strain

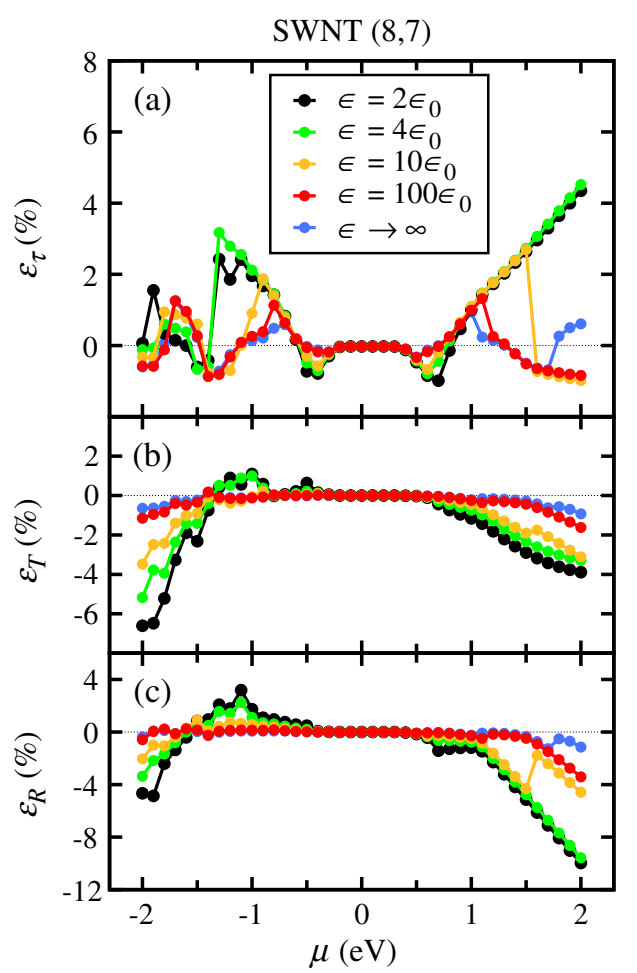

FIG. 4. Fermi-energy dependence of the (a) torsional, (b) axial, and (c) radial strains for the $(8,7)$ SWNT considering different values for the dielectric constant $\epsilon$. Here, $\epsilon \rightarrow \infty$ represents the results obtained when the Coulomb repulsion energy between the electrons is not taken into consideration. 
significantly, the results shown in Figs. 4(a)-4(c) demonstrate a very different behavior. In fact, the electron-electron Coulomb repulsion effect enhances the compression tendency for both $\varepsilon_{T}$ and $\varepsilon_{R}$, and this repulsion actually has a strong influence on $\varepsilon_{\tau}$.

By comparing these results for $\epsilon=4 \epsilon_{0}$ with those obtained for $\epsilon \rightarrow \infty$ (without electron-electron repulsion), it can be seen in Fig. 4(a) that the curved shape of $\varepsilon_{\tau}$ as a function of $\mu$ remains approximately the same. The abrupt changes that appear whenever $\mu$ is close to a subband extremum are also observed for $\epsilon=4 \epsilon_{0}$. However, the magnitude of the total strain is amplified, and the maximum strain occurs at higher values of $|\mu|$. Another important difference is that, while for $\epsilon \rightarrow \infty$ the torsional strain behavior is nearly symmetric with regards to positive and negative values of $\mu$, for $\epsilon=4 \epsilon_{0}$ the symmetry for $+\mu$ and $-\mu$ is broken. The reason for this broken symmetry lies in the fact that, when electron-electron repulsion is considered, the dependence of the torsional strain is strongly coupled to both the radial and the axial deformations, which have asymmetric behaviors with respect to $+\mu$ and $-\mu$, as seen in Figs. 1(d) and 1 (g) for the $(8,7)$ SWNT. In addition, an interesting effect that arises from these new results is that for $\epsilon=4 \epsilon_{0}$, and also for $\epsilon=2 \epsilon_{0}$, the torsional strain $\epsilon_{\tau}$ seems to acquire a practically linear behavior for $\mu$ between 1.0 and $2.0 \mathrm{eV}$, which could be useful for applications in nanoelectromechanical devices.

Furthermore, in Figs. 4(b) and 4(c), we show the dependence of $\varepsilon_{T}$ and $\varepsilon_{R}$ on $\mu$ for different values of the dielectric constant $\epsilon$. In the case of $\epsilon=4 \epsilon_{0}$, we clearly see that there is an enhancement in the compression tendency for both the axial $\varepsilon_{T}$ and the radial $\varepsilon_{R}$ strains as $|\mu|$ increases. In addition, it seems that $\varepsilon_{T}$ acquires a smooth behavior for $\mu$ between +1.0 and $+2.0 \mathrm{eV}$, and $\varepsilon_{R}$ acquires a practically linear behavior for values of $\mu$ between +1.0 and $+2.0 \mathrm{eV}$, similarly to what occurs for $\varepsilon_{\tau}$.

The explanation for these unexpected behaviors of $\varepsilon_{T}$ and $\varepsilon_{R}$ is that, although Eq. (2) predicts a tendency for the nanotube expanding in both the axial and radial directions due to the dependence of $E_{\text {total }}^{\text {(rep) }}$ on the nanotube length $L$ and diameter $d$, it can also be seen that $E_{\text {total }}^{\text {(rep) }}$ depends quadratically on the total nanotube net charge $Q=\lambda L$. Thus, it seems that it is more energetically favorable for the nanotube to compress itself both radially and axially in order to avoid a charge transfer, thus diminishing the

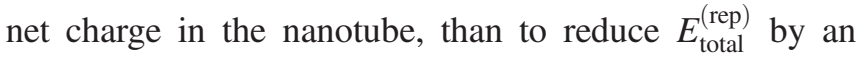
expansion of the nanotube. In fact, the simultaneous compression in both the radial and axial directions causes an increase in the tight-binding hopping energy between different atoms, causing an overall increase in the energy difference between the valence and conduction bands. These changes cause the energy bands to move away from the Fermi energy $\mu$, thus reducing the net charge transferred into the nanotube. As a result, the net charge value observed for $\epsilon=4 \epsilon_{0}$ can be up to $70 \%$ smaller than the values obtained for when the electron-electron Coulomb repulsion was neglected $(\epsilon \rightarrow \infty)$. Also, the same reasoning can be used to explain the surprising influence that the Coulomb repulsion has on $\varepsilon_{\tau}$. In conclusion, the insertion of the Coulomb repulsion causes the nanotube to relax itself in such a way that it minimizes the charge transfer between the nanotube and the reservoir, causing enhanced strains in the axial, radial, and torsional directions, as compared to the case when the Coulomb repulsion is neglected.

Finally, we comment on how the nanotube deformations change for varying effective dielectric constants $\epsilon$. As can be seen in Fig. 4, as the values of $\epsilon$ increase, the contribution from the electron-electron Coulomb repulsion to the total energy decreases, such that the behavior of the axial, radial, and torsional deformations evolves towards the results obtained when the electron-electron repulsion is neglected $(\epsilon \rightarrow \infty)$. Similar results are obtained for the $(9,7)$ and $(9,6)$ nanotubes (not shown here).

In summary, we have applied an extended tight-binding approach for evaluating the Fermi-energy dependence of the structural deformation of SWNTs. We have shown that the nanotube deformation occurs in such a way as to avoid a net charge from being accumulated on the nanotube. This result is understood in view of the fact that the extra charge added to the nanotube increases the total electronic energy, and some of this extra energy can be relaxed by giving rise to additional nanotube axial, radial, and torsional strains. In spite of the simplicity of the model, we can argue that there is a general tendency that the strains which are introduced are such that they minimize the net charge on the nanotube. To verify this, we further consider the contribution of the electron-electron Coulomb repulsion to the total energy by using an effective regularized potential energy model. We show that the inclusion of the Coulomb repulsion leads to larger strains and smaller net charges for the same value of Fermi energy. Torsional strains of up to $\varepsilon_{\tau} \sim 2 \%$ are calculated for a Fermi energy of $\mu=1 \mathrm{eV}$. We would also like to stress that the nearly linear behavior of the torsional strain as a function of the Fermi energy that is observed for some ranges of $\mu$ indicates that this process could be used for the development of high-precision nanoelectromechanical devices, for which the magnitude of the nanotube deformation could be well controlled by the applied gate voltage. Finally, it is important to comment that nanoelectromechanical devices are not the only systems for which the nanotube Fermi energy is fixed by its interaction with a charge reservoir. For example, in the case of carbonnanotube-based nanocomposites, the Fermi energy of the system is mainly controlled by the properties of the matrix material [30]. In such cases, a significant amount of strain is expected on the embedded carbon nanotubes.

A. G. S.F. and E. B. B. acknowledge funding from the Brazilian agency CNPq (Grant No. 307317/2010-2 and INCT NanoBioSimes) and Fundaço Cearense de Apoio 
ao Desenvolvimento Científico e Tecnolgico (FUNCAP) through PRONEX (Grant No. PR2-0054-00022.01.00/11), PRONEM, and CNPq-MIT grant. M. S. D. acknowledges support from NSF (Grant No. DMR-1004147).

[1] B. Witkamp, M. Poot, and H. S. J. van der Zant, Bending-mode vibration of a suspended nanotube resonator, Nano Lett. 6, 2904 (2006).

[2] P. Kim and C. M. Lieber, Nanotube nanotweezers, Science 286, 2148 (1999).

[3] P. Poncharal, Z. L. Wang, D. Ugarte, and W. A. de Heer, Electrostatic deflections and electromechanical resonances of carbon nanotubes, Science 283, 1513 (1999).

[4] J. C. Meyer, M. Paillet, and S. Roth, Single-molecule torsional pendulum, Science 309, 1539 (2005).

[5] V. Sazonova, Y. Yaish, H. Üstünel, D. Roundy, T. A. Arias, and P. L. McEuen, A tunable carbon nanotube electromechanical oscillator, Nature (London) 431, 284 (2004).

[6] H. B. Peng, C. W. Chang, S. Aloni, T. D. Yuzvinsky, and A. Zettl, Ultrahigh frequency nanotube resonators, Phys. Rev. Lett. 97, 087203 (2006).

[7] Y. N. Gartstein, A. A. Zakhidov, and R. H. Baughman, Mechanical and electromechanical coupling in carbon nanotube distortions, Phys. Rev. B 68, 115415 (2003).

[8] G. Sun, J. Kürti, M. Kertesz, and R. H. Baughman, Variations of the geometries and band gaps of single-walled carbon nanotubes and the effect of charge injection, J. Phys. Chem. B 107, 6924 (2003).

[9] L. Pastewka, P. Koskinen, C. Elsasser, and M. Moseler, Understanding the microscopic processes that govern the charge-induced deformation of carbon nanotubes, Phys. Rev. B 80, 155428 (2009).

[10] M. Verissimo-Alves, B. Koiller, H. Chacham, and R. B. Capaz, Electromechanical effects in carbon nanotubes: Ab initio and analytical tight-binding calculations, Phys. Rev. B 67, 161401 (2003).

[11] A. Z. Hartman, M. Jouzi, R. L. Barnett, and J. M. Xu, Theoretical and experimental studies of carbon nanotube electromechanical coupling, Phys. Rev. Lett. 92, 236804 (2004).

[12] R. H. Baughman, C. Cui, A. A. Zakhidov, Z. Iqbal, J. N. Barisci, G. M. Spinks, G. G. Wallace, A. Mazzoldi, D. De Rossi, A. G. Rinzler, O. Jaschinski, S. Roth, and M. Kertesz, Carbon nanotube actuators, Science 284, 1340 (1999).

[13] S. Gupta, M. Hughes, A. H. Windle, and J. Robertson, Charge transfer in carbon nanotube actuators investigated using in situ raman spectroscopy, J. Appl. Phys. 95, 2038 (2004).

[14] J. D. W. Madden, J. N. Barisci, P. A. Anquetil, G. M. Spinks, G. G. Wallace, R. H. Baughman, and I. W. Hunter,
Fast carbon nanotube charging and actuation, Adv. Mater. 18, 870 (2006).

[15] I.-W. P. Chen, Z. Liang, B. Wang, and C. Zhang, Chargeinduced asymmetrical displacement of an aligned carbon nanotube buckypaper actuator, Carbon 48, 1064 (2010).

[16] D. G. Vercosa, E. B. Barros, A. G. Souza Filho, J. Mendes Filho, G. G. Samsonidze, R. Saito, and M. S. Dresselhaus, Torsional instability of chiral carbon nanotubes, Phys. Rev. B 81, 165430 (2010).

[17] K. Kato, T. Koretsune, and S. Saito, Twisting effects on carbon nanotubes: A first-principles study with helical symmetry operations, J. Phys. Conf. Ser. 302, 012007 (2011).

[18] N. Lazic, T. Vukovic, G. Volonakis, I. Miloevic, S. Logothetidis, and M. Damnjanovic, Natural torsion in chiral single-wall carbon nanotubes, J. Phys. Condens. Matter 24, 485302 (2012).

[19] D.-B. Zhang, T. Dumitrica, and G. Seifert, Helical nanotube structures of MoS2 with intrinsic twisting: An objective molecular dynamics study, Phys. Rev. Lett. 104, 065502 (2010).

[20] D.-B. Zhang, E. Akatyeva, and T. Dumitrica, Bending ultrathin graphene at the margins of continuum mechanics, Phys. Rev. Lett. 106, 255503 (2011).

[21] D. Porezag, T. Frauenheim, T. Köhler, G. Seifert, and R. Kaschner, Construction of tight-binding-like potentials on the basis of density-functional theory: Application to carbon, Phys. Rev. B 51, 12947 (1995).

[22] G. G. Samsonidze, Ph.D. thesis, MIT, 2007.

[23] E. B. Barros, A. Jorio, G. G. Samsonidze, R. B. Capaz, A. G. Souza Filho, J. Mendes Filho, G. Dresselhaus, and M. S. Dresselhaus, Review on the symmetry-related properties of carbon nanotubes, Phys. Rep. 431, 261 (2006).

[24] D. M. Himmelblau, Applied Nonlinear Programming (McGraw-Hill, New York, 1972).

[25] T. G. Kolda, R. M. Lewis, and V. Torczon, Optimization by direct search: New perspectives on some classical and modern methods, SIAM Rev. 45, 385 (2003).

[26] L. Yang and J. Han, Electronic structure of deformed carbon nanotubes, Phys. Rev. Lett. 85, 154 (2000).

[27] R. Loudon, One-dimensional hydrogen atom, Am. J. Phys. 27, 649 (1959).

[28] T. G. Pedersen, Variational approach to excitons in carbon nanotubes, Phys. Rev. B 67, 073401 (2003).

[29] R. Langlet, M. Arab, F. Picaud, M. Devel, and C. Girardet, Influence of molecular adsorption on the dielectric properties of a single wall nanotube: A model sensor, J. Chem. Phys. 121, 9655 (2004).

[30] E. Kymakis, I. Alexandrou, and G. A. J. Amaratunga, High open-circuit voltage photovoltaic devices from carbon-nanotube-polymer composites, J. Appl. Phys. 93, 1764 (2003). 